\title{
A Tie-Over Dressing Using a Silicone Tube to Graft Deep Wounds
}

\author{
Cem Inan Bektas, Yuksel Kankaya, Kadri Ozer, Ruser Baris, Ozlem Colak Aslan, Ugur Kocer \\ Plastic, Reconstructive and Aesthetic Surgery Clinic, Ankara Training and Research Hospital, Ankara, Turkey
}

Background The most common cause of skin graft failure is the collection of blood or serous fluid underneath the graft. In our study, we describe the use of silicone tube for tie-over dressing to secure the skin graft margins with the aim of decreasing loss of the skin graft, particularly in grafting of deep wounds.

Methods Between March 2008 and July 2011, we used this technique in 17 patients with skin defects with depths ranging from 3.5 to $8 \mathrm{~mm}$ (mean, $5.5 \mathrm{~mm}$ ). First, the skin graft was sutured with $3 / 0$ silk suture material from its corners. Then, a silicone round drain tube was sutured with $3 / 0$ absorbable polyglactin 910 over the margins of the graft. Finally, long silk threads were tied over the bolus dressing, and the tie-over dressing was completed in the usual fashion.

Results The mean follow-up was 7 months (range, 2-10 months) in the outpatient clinic. Graft loss on the graft margins due to hematoma or seroma was not developed. The results of adhesion between the graft and wound bed peripherally was excellent.

Conclusions In our study, we suggest that use of a silicone tube for additional pressure on the edges of skin grafts in case of reconstruction of deep skin defects.

Keywords Skin transplantation / Occlusive dressings / Silicones
Correspondence: Kadri Ozer

Plastic, Reconstructive and Aesthetic Surgery Clinic, Ankara Training and

Research Hospital, Ankara, Turkey

Tel: +90-312-5953653

Fax: +90-312-3633396

E-mail: kadriozer@hotmail.com

We thank Adile Turan, M.D. (Plastic, Reconstructive and Aesthetic Surgery Clinic, Ankara Training and Research Hospital, Ankara, Turkey) and our nurse, Emel Anayurt, for sharing their valuable time and helpful knowledge.

No potential conflict of interest relevant to this article was reported.

Received: 14 Jun 2013 • Revised: 10 Jul 2013 • Accepted: 20 Jul 2013

pISSN: 2234-6163 • elSSN: 2234-6171 • http://dx.doi.org/10.5999/aps.2013.40.6.711 • Arch Plast Surg 2013;40:711-714

\section{INTRODUCTION}

A skin graft involves completely separating a portion of the epidermis with varying amounts of the underlying dermis from its blood supply and donor-site attachments in order to transfer it to cover to an open wound when direct suture closure is not possible [1]. Skin grafting is used by many surgical specialties in a variety of clinical situations, such as traumatic injuries, burn reconstruction, or surgical wounds. The most common cause of skin graft failure is the collection of blood or serous fluid underneath the graft. Immobilization and the closest possible contact of the skin graft to the wound bed has therefore become an important factor for increasing the success of the skin graft- ing. A commonly used technique to secure immobilization of a skin graft to the wound bed is a tie-over dressing, which is easy to teach to a surgeon-in-training [2]. Although a tie-over dressing is very useful in obtaining pressure in the centre of a skin graft until revascularisation occurs, it does not provide enough pressure and contact in the graft margins and its periphery. That undesirable situation occurs particularly in the skin grafting procedure of deep wounds, which have a difference in height between the skin graft and recipient bed. Therefore, skin graft failure could be visible over the graft margins depending on local hematoma and fluid collection because of less close contact.

Many modifications of the tie-over dressing such as a modified tie-over dressing, stapled tie-over stent, transparent gas bag 
tie-over, tie-over dressing with skin-staples and round rubber bands, tie-over with a sports jacket clip, tie-over dressing with external wire-frame fixation and tension suture technique have been developed to maximize the chance of good adherence of the graft to the recipient [1-5]. In our study, we describe the use of a silicone tube for the tie-over dressing to secure the skin graft margins with the aim of reducing the loss of skin grafts, particularly in the grafting of deep wounds.

\section{METHODS}

The study protocol conformed to the ethics guidelines of the 1975 Declaration of Helsinki, as reflected by the approval of our institution's human research review committee. Between March 2008 and July 2011, we used tie-over dressings with the silicone tube technique in 17 patients who had a deep skin defect that

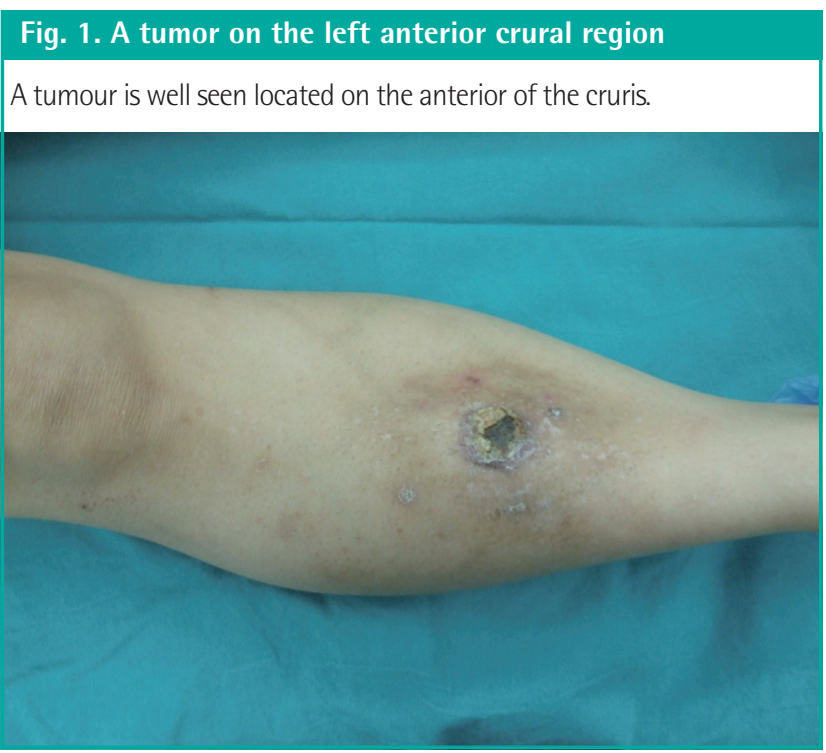

Fig. 2. The defect with a level difference after wide excision A deep skin defect is seen after wide excision of the tumour.

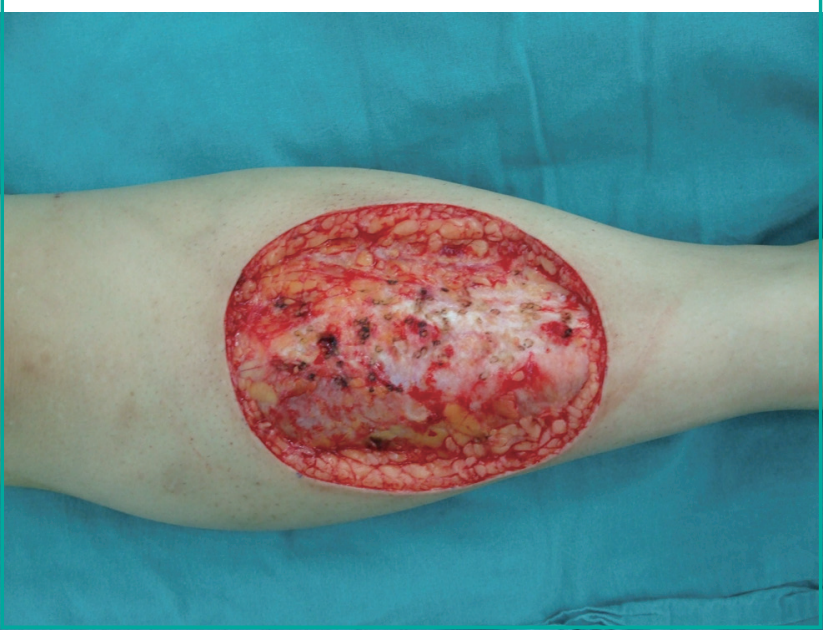

we reconstructed with a skin graft. Their ages ranged from 23 to 65 years (mean, 44 years). Of these 17 patients, the underlying diseases were a burn in 1 patient, skin tumour excision in 9 patients, trauma in 3 patients and pressure sores in the remaining 4 patients (Fig. 1). In all of the patients, the wound edge depth was measured by a ruler and a cotton tip applicator which was lined up perpendicular to the wound from the visible skin of the wound margin to the wound bed nearest to the wound edge. Four designated spots along different sides were measured according to the clock method: The deepest visible part of the wound edge was first measured and called the $12: 00$ position and from that reference point, positions at 03:00, 06:00, and 09:00 were measured with the same method. The average of four spot measurements gave us one unique data point to compare the depths of each skin defect quantitatively. The depth of

\section{Fig. 3. The silicone tube over skin graft margins}

After skin grafting, a silicone tube was placed on the over the wound margin and skin graft.

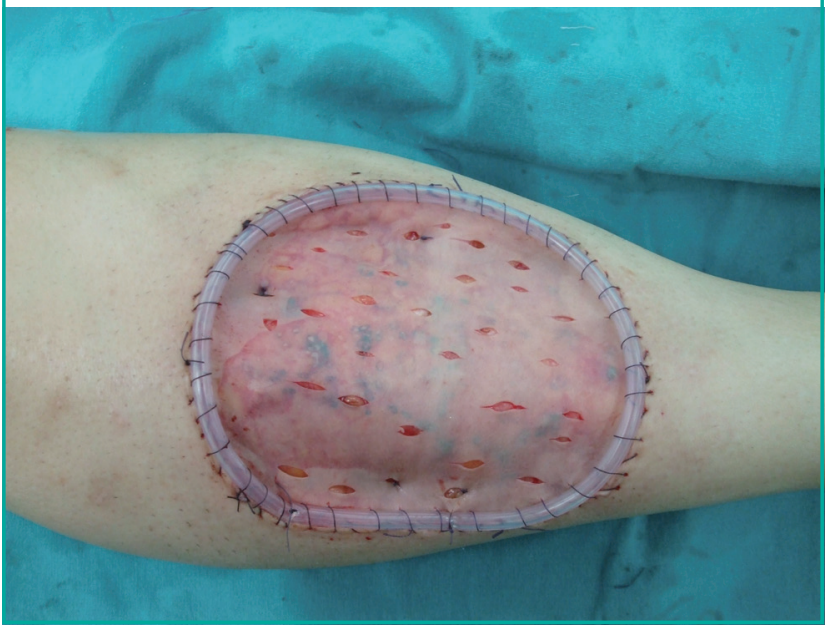

Fig. 4. The tie-over dressing with the silicone tube Traditional tie-over dressing was applied over the skin graft.

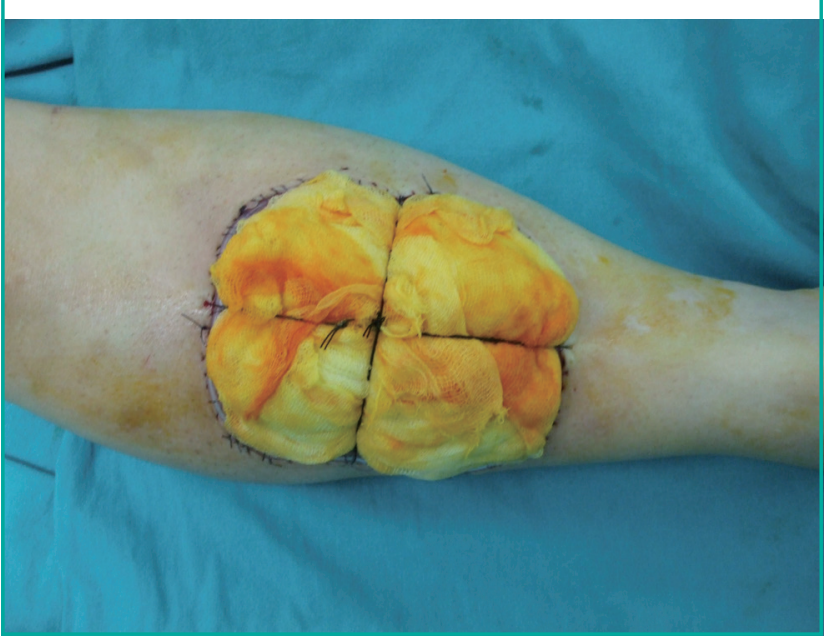


the skin defect margins in our study ranged from 3.5 to $8 \mathrm{~mm}$ (mean, $5.5 \mathrm{~mm}$ in depth).

After the wound was prepared (Fig. 2) and adequate hemostasis was obtained, the skin grafting procedure was performed in the usual manner. A split thickness skin graft was harvested in all of the patients from the posterolateral thigh. First, a skin graft was sutured with $3 / 0$ silk suture material from its corners with equal distances to the wound bed (Fig. 3). Then, a silicone round drain tube was cut as long as the circumference of the wound bed and it was sutured with $3 / 0$ absorbable polyglactin 910 over the margins of the graft (Fig. 4). Suturing was applied continuously as a loop over the silicone tube and margins of the skin grafting area at a suitable distance. The two free edges of the silicone tube were tied with the same suture. Non-adherent petrolatum gauze and cotton was then used as a bolus over it. No

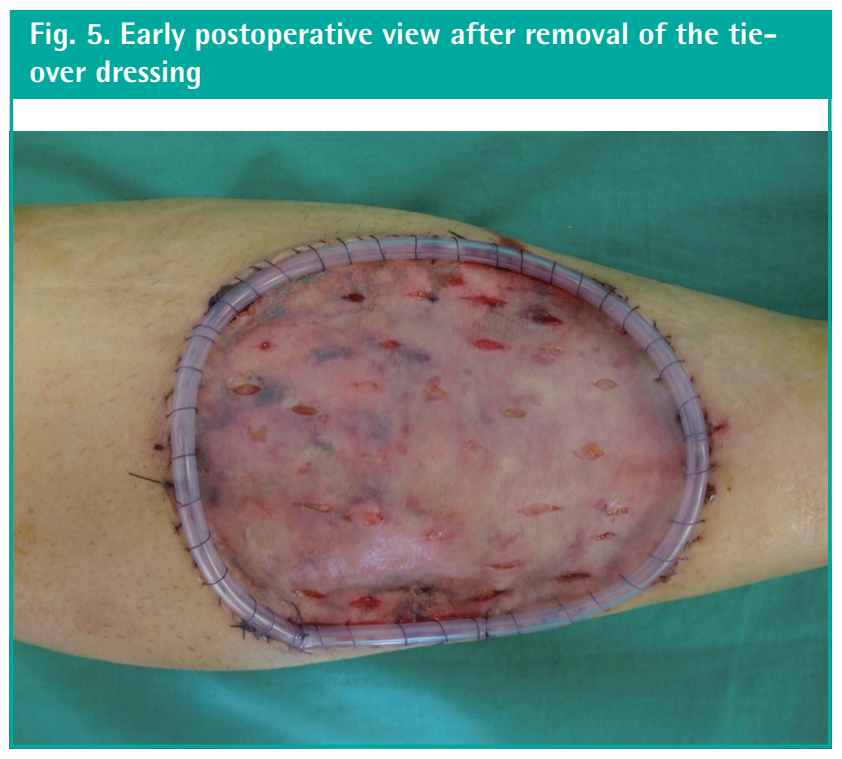

Fig. 6. Good adhesion of the skin graft margins

Good adhesion of the skin graft in particular margins of the skin graft is seen.

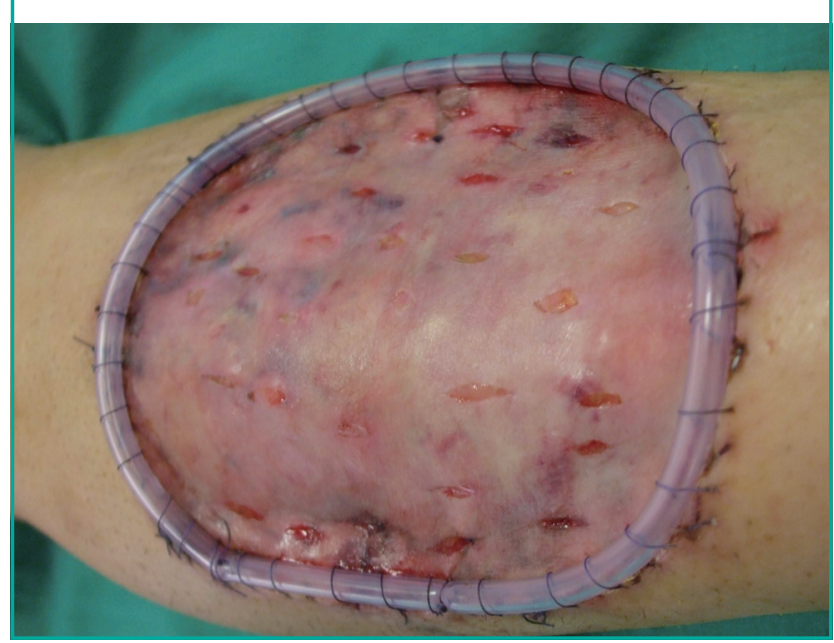

additional suture was performed. Finally, long silk threads were tied over the bolus dressing and thus the tie-over dressing was completed in the usual fashion.

The tie-over dressing with the silicone tube was left in place for about 5 days (range, 2-7 days). The silicone tube was removed at the same time as the removal of the tie-over dressing without any anaesthesia. The graft condition was assessed at the time of the removal of the tie-over dressing and subsequently at the routine follow-up. The mean follow-up was 17 months (range, 12-20 months) in the outpatient clinic.

\section{RESULTS}

Of the 17 patients, 2 patients required reoperation because of

Fig. 7. The posperative skin graft margins after removal of the silicone tube

Good adhesion of the skin graft margins is seen.

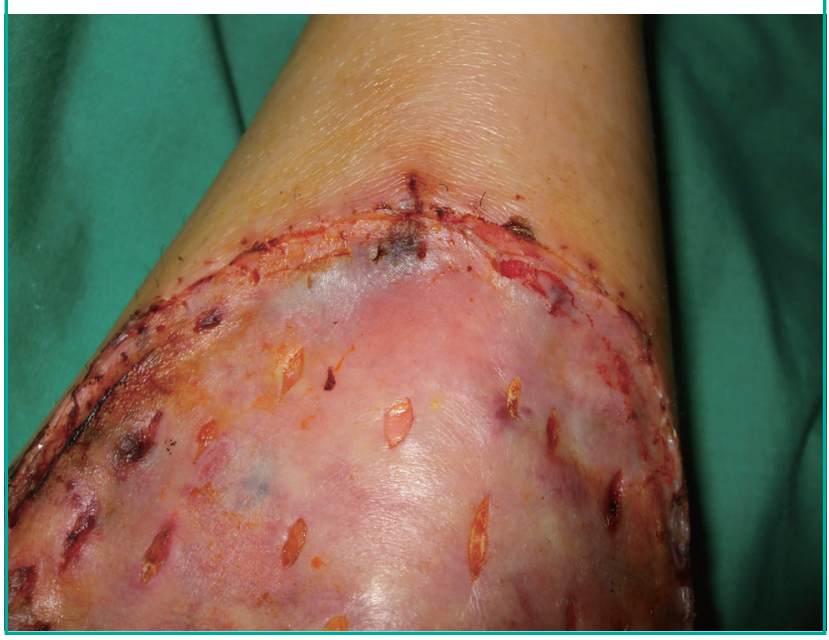

Fig. 8. A late postoperative view showing good margins

Late postoperative view of the skin grafting in deep wounds without any necrosis or failure is seen.

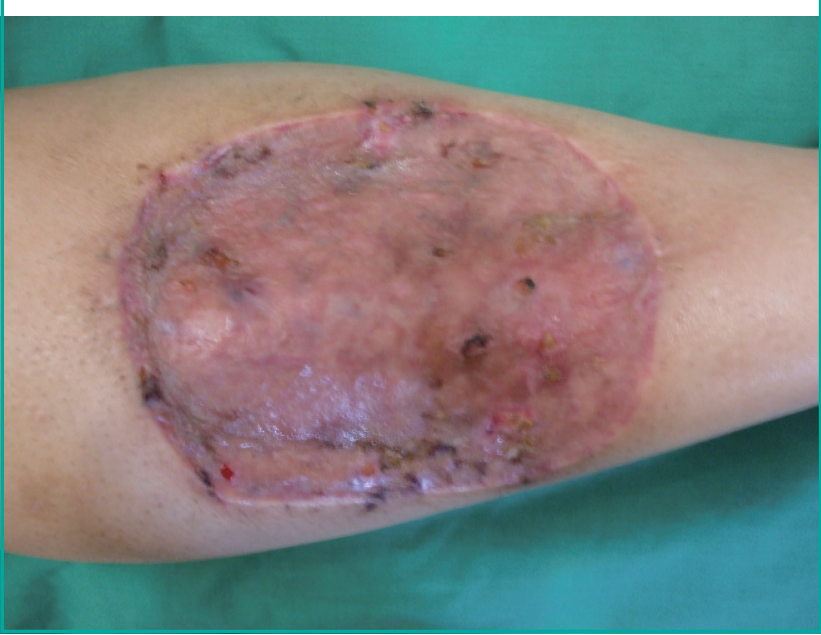


local infections due to inadequate debridement of a traumatic wound. Other than that, no complications were observed. Graft loss on the graft margins due to hematoma or seroma did not develop. The results of adhesion between the graft and wound bed peripherally was excellent (Figs. 5-8).

\section{DISCUSSION}

Securing a skin graft to the recipient wound bed is necessary to prevent complications such as seroma and hematoma, and firm attachment is one of the most important factors for successful skin grafting. A traditional tie-over dressing is still a widely used graft closure method, because it is low in price, easy to use, and easy to teach another surgeon. It is very capable of producing direct pressure over the graft to keep it in contact with its recipient bed. To maximize its effectiveness, many modifications of the tie-over dressing with the use of clips, staples, rubber, k-wires, and so on have been developed [1-5]. Additionally, many alternatives to the tie-over dressing such as a continuous vacuum system, which is more expensive and difficult to access, have been performed [6].

We observed that the soft silicone tube enforced homogeneous pressure over the entire edges of the skin graft and prevented the grafts from lifting at the edges. Due to that pressure, securing the graft edges to its base became easier to accomplish. In our cases, no local hematoma, seroma or graft failure was seen over the graft edges. By removing the sutures on average on the fifth postoperative day, both scar formation and local reactions to the suture material were prevented. By this method, necessity of additional suturing to the edges to keep the graft in contact with the recipient bed was decreased compared with the clas- sical tie-over method. This would shorten the operation time and minimize the suture marks over the skin. The thickness of the silicone tube can be selected according to the depth of the wound margin. One additional advantage of a silicone tube is the feature of ease of application compared to a wire frame [5].

In our study, we recommend the use of silicone tubing for additional pressure on the edges of skin grafts in the reconstruction of deep skin defects. Using the silicone tube with the tieover dressing also shortens the operation time, prevents local complications such as hematoma, seroma or graft loss and minimizes suture mark formation.

\section{REFERENCES}

1. Ergen D, Tan O, Bayindir O. Tension suture technique for skin graft fixation: a novel alternative to tie-over dressing. Burns 2006;32:778-9.

2. Cheng LF, Lee JT, Chou TD, et al. Experience with elastic rubber bands for the tie-over dressing in skin graft. Burns 2006;32:212-5.

3. Kim YO, Lee SJ, Park BY, et al. The tie-over dressing using skin-staples and round rubber bands. Br J Plast Surg 2005;58:751-2.

4. Budi S, Rados J, Stanec Z. A sports jacket clip: a simple method of securing tie-over dressings. J Plast Reconstr Aesthet Surg 2009;62:e495-6.

5. Hirai T, Hyakusoku H, Fumiiri M. The use of a wire frame to fix grafts externally. Br J Plast Surg 1991;44:69-70.

6. De Gado F, Chiummariello S, Monarca C, et al. Skin grafting: comparative evaluation of two dressing techniques in selected body areas. In Vivo 2008;22:503-8. 